\title{
1 Liquid-crystalline lipid phase transitions in lipid droplets selectively remodel the LD
} 2 proteome

4 Authors: Sean Rogers ${ }^{1 *}$, Long Gui ${ }^{1 *}$, Anastasiia Kovalenko ${ }^{1 *}$, Evan Reetz ${ }^{1}$, Daniela Nicastro ${ }^{1}$, 5 W. Mike Henne $e^{1, \#}$

\section{Affiliations:}

$8{ }^{1}$ Department of Cell Biology, UT Southwestern Medical Center, Dallas TX 75390, USA

9 *these authors contributed equally to this work

10 "correspondence should be sent to: mike.henne@utsouthwestern.edu

\section{Summary:}

13 Lipid droplets (LDs) are reservoirs for triglycerides (TGs) and sterol-esters (SEs). How lipids are 14 organized within LDs and influence the LD proteome remains unclear. Using in situ cryo15 electron tomography, we show that glucose restriction triggers lipid phase transitions within LDs generating liquid-crystalline lattices inside them. Mechanistically, this requires TG lipolysis,

17 which alters LD neutral lipid composition and promotes SE transition to a liquid-crystalline phase. Fluorescence imaging and proteomics further reveal that LD liquid-crystalline lattices selectively remodel the LD proteome. Some canonical LD proteins including Erg6 re-localize to the ER network, whereas others remain on LDs. Model peptide LiveDrop also redistributes from

21 LDs to the ER, suggesting liquid-crystalline-phases influence ER-LD inter-organelle transport.

22 Proteomics also indicates glucose restriction elevates peroxisome lipid oxidation, suggesting TG

23 mobilization provides fatty acids for cellular energetics. This suggests glucose restriction drives

24 TG mobilization, which alters the phase properties of LD lipids and selectively remodels the LD 25 proteome.

\section{Key words:}

28 triglyceride; sterol-ester; liquid-crystalline layers; phase transition; endoplasmic reticulum; cryo29 electron tomography; cryo-focused ion beam milling 


\section{Introduction}

Lipid droplets (LDs) are unique endoplasmic reticulum (ER)-derived organelles dedicated to the storage of energy-rich neutral lipids. Structurally LDs are composed of a hydrophobic core of triglycerides (TGs) and sterol-esters (SEs) that is surrounded by a phospholipid monolayer that either contains or is decorated by specific proteins. Beyond their roles in energy homeostasis, recent work highlights the roles of LDs in signaling, development, and metabolism (Welte and Gould, 2017), (Olzmann and Carvalho, 2019), (Walther et al., 2017). These diverse jobs are largely dictated by the LD proteome, but a pervasive question is how specific proteins are targeted to the LD surface. Furthermore, whether the LD proteome is static or dynamic, and how metabolic cues influence LD protein residency is poorly understood.

LDs are generated at the ER and often remain connected to the ER bilayer for extended periods (Jacquier et al., 2011), (Kassan et al., 2013). As such, Type I LD proteins can translocate between the ER and LD monolayer via lipidic bridges connecting the two organelles (Wilfling et al., 2013). Elegant in vitro studies have suggested that LD localization promotes energetically favorable conformational changes within some proteins, and the movement of proteins to LDs from the ER network can even influence their enzymatic activities, or modulate their degradation (Caillon et al., 2020), (Chorlay and Thiam, 2020), (Leber et al., 1998),

51 (Schmidt et al., 2013), (Ohsaki et al., 2006). A second mechanism of LD targeting occurs from 52 the cytoplasm, where soluble proteins insert into the LD monolayer via a hydrophobic region, 53 amphipathic helix, or lipid moiety. Here hydrophobic protein regions recognize packing defects 54 between the phospholipid monolayer lipid head groups, enabling their insertion into the neutral 55 lipid core (Chorlay and Thiam, 2020).

Although monolayer phospholipids can regulate LD protein targeting, how neutral lipids influence protein localization is less understood. However, neutral lipids clearly impact the composition of the LD surface proteome; for example, in yeast, some proteins preferentially decorate TG-rich LDs (Gao et al., 2017). Molecular studies also indicate that protein insertion into the LD neutral lipid core enables proteins to fold with lower free energy, and polar residues within hydrophobic regions can even interact with TG, further anchoring them to the LD (Olarte et al., 2020). However, how neutral lipid pools ultimately influence the composition and dynamics of the LD proteome is relatively unexplored, yet central to our understanding of LD organization and functional diversity.

Neutral lipids generally form an amorphous mixture within the hydrophobic LD core. This organization can change in response to various cellular stimuli. HeLa cells induced into mitotic 
arrest or starvation exhibit lipid phase transitions within their LDs, generating liquid-crystalline lattices (LCLs) inside LDs with a striking onion-like appearance by cryo-electron tomography (cryo-ET) (Mahamid et al., 2019). Yeast biochemical studies also proposed similar segregation of TGs and SEs into discrete layers within LDs (Czabany et al., 2008). This lipid reorganization is attributed to the biophysical properties of SEs, which can transition from disordered to

72 ordered smectic phases under physiological conditions (Kroon, 1981), (Ginsburg et al., 1984),

73 (Shimobayashi S, 2019), (Czabany et al., 2008). Such phase transitions are also associated 74 with human pathologies including atherosclerosis, and liquid-crystalline LDs were even 75 observed in the macrophage of a patient with Tangier disease (Lundberg, 1985), (Katz et al., 76 1977). How these phase transitions are triggered, however, and whether they influence organelle physiology, or are simply a biophysical consequence of the properties of SEs, is unknown.

Here, we utilized budding yeast to dissect the metabolic cues governing lipid phase transitions within LDs. We used cryo-ET of cryo-focused ion beam (cryo-FIB) milled yeast cells to study the in situ architecture of LDs in their native environment, under ambient or glucosestarved conditions. We show that in response to acute glucose restriction, yeast initiate TG lipolysis, which induces the formation of LCLs within LDs. In line with this lipid mobilization, global proteomics reveals that glucose restriction promotes metabolic remodeling favoring peroxisome fatty acid oxidation and mitochondrial metabolism. Furthermore, we find LD liquidcrystalline remodeling selectively changes the LD surface proteome, promoting the redistribution of some proteins from the LD surface to the ER network while others are retained on LCL-LDs.

Results:

92 Acute glucose restriction promotes TG lipolysis-dependent liquid-crystalline phase transitions in LDs

Previous studies from our group indicated that budding yeast exposed to acute glucose restriction (AGR), where yeast are transferred from a glucose-rich (2\%) synthetic complete media to a low-glucose $(0.001 \%)$ media, exhibit metabolic remodeling that favors the production

97 of SEs, which are stored in LDs (Rogers et al., 2021). We used cryo-ET to investigate if AGR 98 also impacts LD morphology. We rapidly froze yeast cells that were either in logarithmic (log99 phase) growth in glucose-rich media, or exposed to $4 \mathrm{hrs}$ of AGR, and used cryo-FIB milling to 100 generate $100-200-n m$-thick lamellae of the vitrified cells. These lamellae were then imaged by 
101 cryo-ET to reveal the three-dimensional (3D) structure of native LDs in situ. The cryo-FIB milled

102 lamella exhibited a well-preserved yeast ultrastructure, including the nucleus, vacuole, 103 mitochondria and LDs (Figure 1A, SFigure1, SMovie 1). Typical LDs could be distinguished 104 from other cellular organelles by their relatively electron-dense, amorphous interior that was 105 surrounded by a thin phospholipid monolayer (Figure 1B, SMovie 2). In contrast to normal LDs 106 in glucose-fed log-phase cells, $77 \%$ of the LDs observed in 4hrs AGR-treated yeast displayed 107 reorganization of their interior, including the appearance of distinct concentric rings in the LD 108 periphery (Figure $\mathbf{1}$ C-D, M for quantification, SMovie 3). These rings appear similar to 109 lattices previously observed in liquid-crystalline-phase LDs, which exhibited a regular spacing of $110 \sim 3.4-3.6 \mathrm{~nm}$ between their layers, suggesting they were composed of sterol-esters (Mahamid et 111 al., 2019), (Engelman and Hillman, 1976). Indeed, our line-scan analysis showed a regular

$1123.4 \mathrm{~nm}$ spacing between rings (Figure 1E), suggesting these LDs exhibited liquid-crystalline 113 lattices (LCLs). Thus, we refer these "onion-like" LDs as LCL-LDs. Notably, these were never 114 observed in the log-phase yeast (Figure 1B, M).

115 In addition to the peripheral lattices, the amorphous center of LCL-LDs was unusually 116 sensitive to electron radiation, causing excessive radiolysis and "bubbling" (i.e. the generation of 117 a gas bubble trapped in the ice that appears white in cryo-EM images) during tilt-series 118 acquisition (Figure 1C, white arrow). This increased radiation sensitivity was only observed in 119 LCL-LDs, but not in LDs with entirely amorphous lumen (i.e. not observed in the $23 \%$ unordered 120 LDs of AGR-treated yeast, nor in any LDs of log-phase yeast). We generated comparative 121 'bubblegrams', (i.e. a series of 2D cryo-EM images where the same sample area was exposed 122 to an increasing amount of electron dose), which revealed that the centers of LCL-LDs exhibited 123 bubbling following exposure to $<30 \mathrm{e} / \AA^{2}$, whereas amorphous LDs from log-phase yeast did not 124 show any bubbling even at 400 e/ $/ \AA^{2}$ dosages (SFigure1 A-J). Previous studies of electron 125 radiation-induced bubbling of frozen biomolecules in aqueous solution and cells demonstrated 126 that similar gas bubbles contained mostly molecular hydrogen gas (Leapman and Sun, 1995) 127 (Aronova et al., 2011). Although the mechanism of radiation-induced bubbling and increased 128 radiation-sensitivity within the center of LCL-LDs is not clear, it may be due to the production of 129 gases derived from a specific combination of lipids or metabolites present within LCL-LDs.

130 To investigate the effects of AGR stress on yeast neutral lipid pools, we monitored TG 131 and SE levels in log-phase and 4hrs AGR-treated yeast. Indeed, AGR treated yeast contained 132 significantly less TGs (Figure 1K). As expected, AGR yeast also had increased amounts of SEs

133 (Figure 1K), as previously observed (Rogers et al., 2021), indicating the TG:SE ratio within the 134 LDs was significantly decreased to $\sim 0.5: 1.5$ compared to a normal ratio of $\sim 1: 1$ (Leber et al., 
1994). We hypothesized that LCL-LD formation was promoted by TG loss from LDs. To test

136 this, cryo-ET was performed on yeast lacking the major TG lipases $(\operatorname{tg} / 3,4,5 \Delta)$. Indeed, 4hrs

137 AGR treated $\operatorname{tg} / 3,4,5 \Delta$ yeast did not form any detectable LCL-LDs (Figure 1G, M), suggesting

138 TG lipolysis was required for LCL-LD formation. In support of this, LDs in wildtype (WT) AGR-

139 treated yeast were significantly smaller in diameter than log-phase LDs, and this reduced size

140 was suppressed in $\operatorname{tg} / 3,4,5 \Delta$ yeast (Figure $\mathbf{1 N}$ ), suggesting the size reduction was due to lipid 141 loss via TG lipolysis.

142 To further dissect how TGs influence LCL-LDs, we treated yeast with $0.1 \%$ oleic acid $143(\mathrm{OA})$, which promotes TG synthesis. As expected, OA elevated cellular TG levels in yeast when 144 they were cultured in it during 4hrs AGR treatment (Figure 1L), and notably no LCL-LDs were 145 observed during log-phase nor in this AGR condition (Figure 1F, H, M). In line with this, 146 whereas LD sizes in AGR-treated yeast were significantly smaller than in log-phase cells, their 147 sizes slightly recovered under the AGR plus OA condition (Figure 1F, N). Since we previously 148 observed that the nucleus-vacuole junction (NVJ) can serve as a site for LD biogenesis during 149 nutrient stress (Hariri et al., 2018), we also examined whether NVJ loss impacted LCL-LD 150 formation. Cryo-ET of nvj1s yeast cells showed the expected loss of tight contacts between the 151 outer nuclear envelope and the vacuole (SFigure 1K, L). However, nvj1 $1 \Delta$ yeast exhibited $\sim 75 \%$ 152 LCL-LDs under AGR conditions, indicating that the NVJ was not required for LCL-LD formation 153 (Figure 1I, J, M).

154 Since SEs can form liquid-crystalline lattices, we tested whether SEs were required for 155 LCL-LD formation. We monitored LDs in are1are2s yeast that cannot synthesize SEs. 156 Surprisingly, in 15 different cryo-FIB lamella of are1are2 $\Delta$ yeast cells no LDs could be observed 157 (SFigure 1M). However, fluorescence staining with monodansylpentane (MDH) LD stain 158 confirmed the presence of LDs in are1are2 $\Delta$ yeast during AGR stress, but they were small and 159 sparse in many yeast compared to any of the other examined strains (SFigure 1N). The 160 reduction in LD size and abundance may account for the inability to observe LDs in the cryo161 tomograms of the $100-200 \mathrm{~nm}$ thick lamellae.

162 Collectively, these data suggest that TG abundance is a key modulator of the SE phase 163 transitions within the LD, and indicate Tgl-dependent TG lipolysis during AGR promotes LCL-LD 164 formation by depleting the TG pool that maintains SE in its disordered phase. 
While studies indicate that LD proteins may interact with TGs contained within the LD interior (Olarte et al., 2020), (Santinho et al., 2021), it is unknown whether smectic lipid phase transitions influence LD protein targeting. Therefore, we imaged the canonical LD protein Erg6 tagged with mNeonGreen (Erg6-mNg) over time in AGR conditions. As expected, Erg6-mNg initially colocalized with LD stain at the start of $A G R(t=0)$. However, the Erg6 labeling pattern

172 changed after $\sim 1 \mathrm{hr}$ AGR, and primarily decorated the cortical ER and nuclear envelope (Figure

173 2A). Erg6-mNg remained at the ER network throughout 2, 4, and $24 \mathrm{hrs}$ AGR, and notably the

174 LD stain gradually dimmed over these time-points, consistent with the loss of LD volume via

175 lipolysis. Remarkably, the addition of $0.1 \%$ OA, or genetic ablation of TG lipases both rescued

176 Erg6-mNg LD targeting at 4hrs AGR (Figure 2B, SFigure 2A). Since our cryo-ET results

177 showed lack of LCL-LD formation in these conditions, it suggested that Erg6-mNg de-

178 localization from LDs tightly correlates with LCL-LD formation.

179 To more directly test whether the biophysical properties of LD lipids influenced Erg6-

$180 \mathrm{mNg}$ localization, rather than other metabolic changes attributed to AGR stress, we briefly

181 heated Erg6-mNg expressing yeast after 4hrs AGR to $40^{\circ} \mathrm{C}$, which is above the predicted phase

182 transition temperature for smectic-phase SEs. Indeed, Erg6-mNg significantly, although not

183 fully, re-localized from the ER network to LDs after only 15 minutes at $40^{\circ} \mathrm{C}$ (Figure 2B). To

184 quantify the extent of Erg6-mNg LD localization, we calculated its relative Manders M1

185 coefficient, which measures total Erg6-mNg signal that overlaps with LD marker MDH. 4hrs

186 AGR stress was accompanied by an $~ 75 \%$ decrease in Erg6-mNg positive LDs (Figure 2C). In

187 agreement with imaging, addition of $0.1 \%$ OA returned the M1 coefficient to WT values. Brief

188 heating also significantly, though not fully, increased the M1 coefficient.

189 Next, we investigated whether AGR caused a general de-localization of other canonical

190 LD proteins from LDs. However, PIn1-mNg, a perilipin-like protein also known as Pet10 (Gao et

191 al., 2017), maintained stable LD association following 4hrs AGR, suggesting the de-localization

192 of LD proteins during LCL-LD formation may be selective (Figure 2D, E). Recently, perilipin

193 homo-oligomerization was proposed to contribute to the stable association of perilipins on LDs

194 (Giménez-Andrés et al., 2021). To test whether oligomerization could enhance LD protein

195 targeting during AGR, we artificially oligomerized Erg6 by tagging it with tetrameric DsRed2.

196 Indeed, unlike monomeric Erg6-mNg, Erg6-DsRed2 maintained LD targeting during 4hrs AGR

197 (SFigure 2B). Collectively, this suggests that: 1) LD protein de-localization during AGR-

198 associated LCL-LD formation may be selective for certain proteins, and 2) oligomerization may 199 enhance protein retention on these LDs. 


\section{Imaging known LD proteins reveals their selective retargeting to the ER during AGR}

Given the different targeting patterns of Erg6 and PIn1 in AGR, we next examined the location of other annotated LD proteins by tagging them with mNeonGreen $(\mathrm{mNg})$ and examining them in log-phase and 4hrs AGR-treated yeast. As expected, four known LD proteins Rer2-mNg, Hfd1-mNg, Yeh1-mNg (an LD-localized SE lipase), mNg-Say1 (which is annotated to target both LDs and the ER network), primarily decorated LDs in log-phase yeast. However, after 4hrs AGR all four proteins displayed ER and nuclear envelope localization, and displayed significantly reduced M1 coefficients, like Erg6 (Figure 3A, B). Similarly, Ayr1-mNg (a bifunctional lipase), as well as Anr2-mNg (a LD protein of unknown function predicted to be palmitoylated) also localized to LDs in log-phase yeast, but displayed primarily ER network targeting after 4hrs AGR (SFigure 3A). Collectively, this suggests that similar to Erg6, many canonical LD proteins exhibit more ER localization following AGR exposure, and indicates that LCL-LD formation may alter the protein composition of the LD surface.

Protein movement between the LD and ER compartments has previously been described for Type I LD proteins, which move between the ER and LDs via lipidic bridges connecting them (Wang et al., 2016). Although we observed several proteins that localized more prominently to the ER versus LDs during AGR, whether any of these represented canonical Type I LD proteins was not clear. Therefore, to interrogate whether Type I LD proteins could be re-targeted or retained at the ER during LCL-LD formation, we monitored GFP-tagged LiveDrop (Wang et al., 2016), a minimal model polypeptide for Type I LD proteins, in log-phase and 4hrs AGR-treated yeast. As expected, GFP-LiveDrop localized predominantly to LDs in log-

222 phase yeast, but a dim ER network signal was also detected, consistent with its dual organelle 223 targeting (Figure 3C). In contrast, following 4hrs AGR GFP-LiveDrop was more prominently at 224 the ER network, and its M1 coefficient was significantly decreased (Figure 3C, D). This 225 suggests that AGR and the associated LCL-LD formation promotes Type I LD protein re226 distribution to, or retention at, the ER network versus LDs.

227 Since TG lipases were required for LCL-LD formation in AGR (Figure 1G, M), we next 228 monitored the sub-cellular localization of all Tgl lipases by fluorescence microscopy. As 229 expected, the major TG lipase Tgl3-mNg, as well as Tgl4-mNg (TG lipase) and Tgl1-mNg (SE 230 lipase) all decorated LDs in log-phase yeast (Figure 3E, F). Remarkably, all three proteins 231 retained LD localization following 4hrs AGR, likewise displaying unaltered M1 coefficients 232 (Figure 3E, F). Tgl5-mNg (TG lipase) also displayed LD targeting in both log-phase and 4hrs 233 AGR yeast (SFigure 3B). This suggests that in contrast to several other LD proteins, Tgl lipases 
maintain LD association during AGR, where they locally deplete the LD TG pool, promoting lipid phase transitions within the LD.

\section{Comparative proteomics reveals changes to the LD proteome in AGR stress}

Since fluorescence imaging revealed that several LD proteins change sub-cellular distribution in AGR conditions, we next aimed to comprehensively map how AGR stress alters

240 the LD proteome. We performed LC-MS/MS proteomics on LDs that were isolated from logphase and 4hrs AGR-treated yeast using density gradient centrifugation (Figure 4A). To evaluate the quality of our LD isolation protocol, we performed Western blotting of whole-cell lysates and the subsequent LD isolation fractions. We found a clear de-enrichment of

244 mitochondrial protein Por1 and the abundant plasma membrane protein Pma1 in the LD fractions, suggesting the LD fractions were relatively pure (Figure 4B).

Given that AGR stress likely changes the global abundance of some proteins, we also conducted LC-MS/MS proteomics on the non-LD infranatant fractions generated during LD isolation, as well as whole-cell lysates of yeast in log-phase or 4hrs AGR treatment. We combined these datasets with our isolated LD proteomics to obtain a more robust dataset of high-confidence LD proteins in these conditions. This approach generated an adjusted LD enrichment score, defined as the "LD confidence score". The approach is based on previous work from (Bersuker et al., 2018), and accounts for the spectral abundance of each protein in the LD fraction, while subtracting out the corresponding abundance from the non-LD infranatant fraction. Plotting this LD confidence score (x-axis) as a function of protein whole-cell abundances (y-axis) thus identified candidate proteins that enriched or de-enriched in AGRassociated LD fractions (Figure 4C). For example, proteins that increased in relative abundance in LD fractions during AGR are represented on the right side of the $x$-axis, whereas those that decreased are on the left side. It should be noted that many proteins did not change greatly in overall whole-cell abundance, and are thus are positioned along 0 on the y-axis. As expected, many proteins not normally associated with LDs change little on the $x$-axis, but may change substantially in whole-cell abundance during AGR, and are thus positioned vertically along the 262 y-axis.

As expected, this approach revealed that Erg6 was among the most de-enriched 264 proteins in LD fractions at 4hrs AGR (Figure 4C, left side of plot), whereas PIn1 was one of 265 the most enriched (Figure 4C, right side of plot). Notably the LC-MS/MS detected nearly all 266 annotated LD proteins (Currie et al., 2014), although some of these displayed changes in 267 abundance that appeared different from the localization patterns we observed by fluorescence 
microscopy (SFigure 4A). The reason for these distinctions likely reflects the differences between imaging and biochemical methodologies, as well as some (expected) contamination of the LD fractions with co-purifying ER membranes during the LD isolation.

Using this approach, our proteomics also revealed a subset of proteins that are not

272 annotated to localize to LDs, but were nonetheless detected in high abundance in the isolated

273 LD fractions during AGR stress. This included Iml2 (Figure 4C, right side of plot), which is a

274 sterol-associated protein required for the clearance of protein inclusions, and was previously

275 observed associated with LDs bound to inclusion bodies (Moldavski et al., 2015). To investigate

276 this, we imaged $\mathrm{mNg}$-tagged $\mathrm{Iml}$, revealing that $\mathrm{Iml2}-\mathrm{mNg}$ was throughout the cytoplasm in

277 log-phase yeast, whereas it subtly decorated the nuclear envelope and cortical ER at 4hrs AGR

278 (SFigure 4B). Even though we did not visibly detect Iml2-mNg on LDs, this may be because

279 LDs need to be associated with protein inclusions for $\mathrm{Iml2}$ to visibly enrich on them by

280 fluorescence microscopy (Moldavski et al., 2015).

281 Our proteomics also indicated that two proteins containing Bin/Amphiphysin/Rvs (BAR) 282 domains involved in Golgi/endosomal membrane trafficking, Snx4 and Gvp36, were enriched on

283 LDs following 4hrs AGR (Figure 4C). BAR domains are membrane binding modules, and many

284 BAR proteins contain amphipathic helices or other membrane inserting modules that could, in 285 principle, insert into LDs. Furthermore, BAR protein GRAF1a was previously observed on LDs 286 in human cells (Lucken-Ardjomande Häsler et al., 2014). Indeed, while Snx4-mNg formed 287 cytoplasmic foci not colocalized with LDs in log-phase growth, Snx4-mNg foci did appear co288 localized with a subset of LDs following 4hrs AGR (Figure 4D). In contrast, Gvp36-mNg 289 distributed mostly throughout the cytoplasm in both log-phase and 4hrs AGR stress, and was 290 not detectably enriched on LDs by fluorescence microscopy (SFigure 4B). Collectively, this 291 indicates that AGR stress, which results in SE phase transition and LCL-LD formation, also 292 selectively remodels the LD proteome. The uncoating of canonical proteins from LDs may lead 293 to enhanced LD association of non-canonical factors or membrane trafficking proteins with the 294 phospholipid surface of LDs.

Global proteomics indicates AGR promotes fatty acid oxidation during metabolic 297 remodeling

298 Energy depletion drives metabolic remodeling in yeast, favoring the reorganization of 299 organelles and the utilization of alternative carbon sources when glucose is restricted (Marini et 300 al., 2020) (Eisenberg and Büttner, 2014). Since we conducted whole-cell LC-MS/MS proteomics 301 of log-phase and 4hrs AGR yeast, we next examined these datasets to determine whether 
changes in whole-cell protein abundances revealed patterns of metabolic remodeling that involved LDs and their lipids. Indeed, we found that 4hrs AGR stress induced changes in the abundances of many proteins involved in fatty acid metabolism. In particular, peroxisome enzymes involved in fatty acid oxidation (FAO), including Pot1, Fox2, and Cta1 were among the most increased in abundance during AGR compared to log-phase growth (Figure 4E, right side of plot). Also elevated were the peroxisome-associated fatty acyl-CoA ligase Faa2, the acetyl-CoA transporter Crc1 (which transports acetyl-CoA derived from peroxisome FAO to mitochondria), as well as Yat1, a carnitine acetyl-transferase that works with Crc1 to promote acetyl-CoA utilization within mitochondria. Enzymes related to the tricarboxylic acid cycle including Icl1 and Idp2, the malate synthase Mls1, and acetyl-CoA synthase Acs1 were also among the most elevated proteins in AGR-treated yeast (Figure 4E). In contrast, amino acid transporters like Mup1 and Lyp1 were significantly decreased in abundance (Figure 4E, left side of plot), consistent with their turnover during glucose starvation that promotes adaptive metabolic remodeling (Lang et al., 2014), (Wood et al., 2020).

Collectively, this indicates that glucose restriction promotes the mobilization of TGs from LDs that may provide fatty acids as fuel for cellular energetics in peroxisomes and mitochondria. Indeed, acetyl-CoA generated by peroxisome FAO can be delivered to mitochondria to fuel its energetics in the absence of glucose, suggesting inter-organelle remodeling during glucose restriction that enables LD-derived lipids to ultimately fuel alternative carbon metabolism. An additional consequence of this Tgl-dependent TG mobilization is a shift in the neutral lipid ratios in LDs, ultimately giving rise to SE transition into a liquid-crystalline phase within the LDs.

\section{Discussion}

325 Emerging evidence suggests the phase transition properties of cellular biomolecules, 326 such as proteins in membraneless organelles, directly influence cell physiology and 327 organization. Like proteins, lipids also undergo phase transitions, and can form liquid-crystalline 328 lattices that are observed in human diseases like atherosclerosis, or in organelles like LDs. 329 However, the metabolic cues that drive these phenomena, and their impact on organelle 330 physiology, are unclear. Here we show that in yeast, AGR stress promotes the formation of 331 liquid-crystalline lipid phase transitions within LDs. These transitions require TG lipolysis, 332 suggesting the loss of TG within the hydrophobic core of LDs promotes the transition of SEs 333 from an amorphous to a smectic liquid-crystalline phase. In agreement with this, we find AGR 334 drives metabolic remodelling that elevates peroxisome-mediated lipid oxidation. Furthermore, 335 we provide evidence that LCL-LD phase transitions alter the LD proteome (Figure 4F). 
How proteins are targeted to LDs is still poorly understood, and involves trafficking from the ER network or cytoplasm to the LD surface. In this study, we revealed that the LD proteome dramatically differs between AGR-treatment and log-phase growth. Erg6, a canonical LD protein, relocalizes to or is retained at the ER network, suggesting it moves from LDs to the ER via a lipidic bridge. This LD delocalization appears suppressed or quickly reversed when yeast cells are briefly heated to $40^{\circ} \mathrm{C}$, (i.e. above the predicted melting temperature of smectic-phase SEs), suggesting direct movement of the proteins between LD and ER via ER-LD connections. In line with this, GFP-LiveDrop, which under log-phase conditions targets primarily to LDs, appears predominantly ER localized during AGR. Collectively, this suggests that Type I LD proteins favor ER localization versus the surface of LCL-LDs. This also indicates that many yeast LDs maintain connections to the ER network and thus exhibit the lipidic bridges necessary for this inter-organelle trafficking, consistent with earlier work (Jacquier et al., 2011). The redistribution of LD proteins to the ER may be due to changes in LD monolayer fluidity after LCL-LD formation, which could alter the energetic favorability of proteins to remain on the LD surface. We also cannot rule out that the lipid composition of the ER network changes during AGR to a state that favors protein targeting or retention. We also find that artificially multimerizing Erg6 with a DsRed2 tag promotes its LD retention at AGR, implying protein oligomerization enhances LD retention, as has previously been observed for perilipins (Giménez-Andrés et al., 2021).

Whereas Erg6 delocalized from LDs during AGR, TG lipases Tgl3,4,5 remained LD bound. Although the LD anchoring mechanisms for Tgl lipases are not fully understood, this implies that LDs continue to mobilize TG during AGR, gradually altering the TG:SE neutral lipid ratio in a manner that supports SE phase transition. Indeed, AGR-treated yeast contain less TGs, consistent with lipolysis that provides fatty acids to fuel metabolic energetics. Fatty acids derived from these TGs are likely substrates for peroxisome FAO, of which several key enzymes are elevated during AGR stress. The acetyl-CoA produced from FAO could also fuel mitochondrial energetic pathways, several proteins of which are elevated by proteomics. LCLLDs also exhibited de-targeting of enzymes like Hfd1, Rer2, and Say1. It is possible these 364 enzymes' re-distributions influences their activities, and therefore promote metabolic 365 remodeling. Indeed, several Erg pathway enzymes also appeared de-enriched from LDs during 366 AGR by proteomics, and Erg1 is more active at the ER than on LDs (Leber et al., 1998).

Our proteomic and imaging analysis also revealed that LDs may become decorated with 368 non-LD proteins during AGR stress. This included the BAR domain protein Snx4, which co369 localized with some LDs only during AGR stress. As BAR proteins contain membrane 
binding/inserting modules, it is possible that Snx4 associates with LDs during AGR by inserting into its monolayer surface. Since the LD surface is normally densely coated with proteins, it is also possible Snx4 and other proteins may associate with the LD surface as it is uncoated of canonical LD proteins during AGR stress. Proteomics also detected Iml2 on LDs during AGR. Previous work proposed that Iml2 associated with LDs, and promoted the delivery of sterols to protein inclusions during their clearance in an unknown mechanism involving LDs (Moldavski et al., 2015). Although unclear, it is possible Iml2 may influence sterol metabolism on LCL-LDs.

This study is a significant step toward enhancing understanding how lipid phase transitions influence LD and organelle protein composition and ultimately function. Future studies will interrogate whether such changes in the LD proteome reflect metabolic remodeling that ultimately enable yeast to adapt to glucose shortage.

\section{Materials and Methods}

Please see STAR Methods for a full description of the Methodology.

\section{Acknowledgement}

386 We thank Jonathan Friedman, and members of the Henne and Nicastro labs for helpful insights during this study. We thank Daniel Stoddard for management of the UTSW electron microscope facilities and training, and Gang Fu for some data acquisition. The UT Southwestern CryoElectron Microscopy Facility is supported in part by the CPRIT Core Facility Support Award RP170644. We would also like to thank the UTSW proteomics and live cell imaging facilities for their assistance with data collection and analysis. Finally, we would like to thank Dr. Joel Goodman for the Pln1 antibody. W.M.H. is supported by funds from the Welch Foundation (I1873), the NIH NIGMS (GM119768), NIDDK (DK126887), Ara Parseghian Medical Research

394 Fund, and the UT Southwestern Endowed Scholars Program. S.R. is supported in part by a NIH 395 T32 training grant (5T32GM008297). L.G., E.R., and D.N. are supported by the Cancer 396 Prevention and Research Institute of Texas grant RR140082 to D.N. This research was 397 supported in part by the computational resources provided by the BioHPC supercomputing 398 facility located in the Lyda Hill Department of Bioinformatics, UT Southwestern Medical Center.

\section{Figure Legends}

401 Figure 1: Visualization of the liquid-crystalline layers in lipid droplets (LCL-LD) promoted 402 by TG lipolysis using in situ cryo-ET. A) Representative tomographic slice from a cryo-FIB403 milled and cryo-ET reconstructed wildtype (WT) yeast cell grown for $4 \mathrm{hrs}$ under acute glucose 
restriction (AGR). Note the "bubbled" (lighter) centers of the LDs (L). V, vacuole. N, nucleus. A different tomographic slice of the boxed LD is also shown in (C). B-J) Representative tomographic slices of LDs in yeast from glucose-fed WT in log phase (B), WT after 4hrs AGR (C, boxed area magnified in D, E shows line-scan plot of area between yellow arrowheads), WT

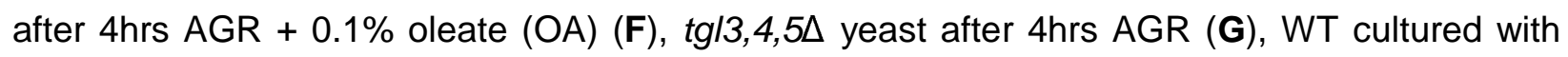
$2 \%$ glucose and $0.1 \% O A(\mathbf{H}), n v j 1 \Delta$ after $4 \mathrm{hrs}$ AGR (I, and boxed area magnified in $\mathbf{J})$. Liquidcrystalline layers (LCL) were only observed in LDs from WT and $n v j 1 \Delta$ yeasts in AGR (C, D, I, J). White arrows highlight the 'bubbles' due to electron radiation in centers of LCL-LDs. K) Quantification of relative whole-cell TGs and SEs in log and 4hrs AGR conditions. L) Relative TGs in log and 4hrs AGR conditions. M, N) \% abundance of LCL-LDs (M) and diameters of LDs (N) under various conditions measured in cryo-tomograms. Note that the observed diameter depends on the plane at which the LDs were sectioned; therefore, for size measurements, only LDs with clearly visible monolayer (indicating a slice through the LD center) were included. Scale bars: 200nm (A). 50nm (B-C, F-I). 20nm (D, J).

Figure 2: Erg6 LD de-localization correlates with LCL-LD formation. A) Yeast expressing Erg6-mNeonGreen $(\mathrm{mNg})$ and stained for LDs (monodansylpentane, MDH) at time-points when yeasts were transferred from log-phase ( $2 \%$ glucose) to acute glucose restriction (AGR). Red arrows indicate protein targeting. B) Yeast with Erg6-mNg and LD/MDH stain in log-phase (2\% glucose), $A G R$, and $A G R+0.1 \%$ oleate $(O A)$, and $A G R+15 \min 40^{\circ} \mathrm{C}$. C) Manders $M 1$ coefficient

424 of Erg6-mNg colocalization with LD stain MDH in various conditions. D) Pln1/Pet10-mNg in log 425 and 4hrs AGR. E) M1 coefficient of PIn1-mNG with LD targeting. Statistics are one-way ANOVA. Scale bars $5 \mu \mathrm{m}$.

Figure 3: Fluorescence imaging reveals selective remodeling of LD proteome during AGR. A) Yeast with mNeongreen ( $\mathrm{mNg}$ )-tagged LD proteins with MDH LD stain in log and 4hrs AGR. B) M1 coefficient of proteins in A. C) Yeast with GFP-LiveDrop and MDH LD stain in log and 4hrs AGR yeast. D) M1 coefficient of proteins in C. E) Yeast with $\mathrm{mNg}$-tagged Tgl1,3,4 and

432 stained with MDH LD marker in log-phase or 4hrs AGR. F) M1 coefficient of proteins in E. Scale 433 bars $5 \mu \mathrm{m}$.

Figure 4: Comparative proteomics indicates non-canonical protein association with LDs, 436 and metabolic remodeling during AGR. A) Schematic of LD isolation protocol. B) Western 437 blot of whole cell lysate (WCL), and fractions of LD isolation protocol as in A. Pma1: plasma 
membrane marker, Por1: mitochondria marker. PIn1: LD marker. Tubulin: cytoplasmic marker C) Plot of protein abundances in whole-cell proteomics (y-axis) versus their change in LD confidence score (see methods for description of this value) Data are average of 4 independent expts. D) Micrographs of Snx4-mNg and LD/MDH stain in log and 4hrs AGR yeast with M1 coefficient of LD colocalization. E) Volcano plot showing $\log _{10} p$-value and $\log _{2}$ abundance

443 changes in whole-cell abundance of proteins in $4 \mathrm{hrs}$ AGR treatment versus $2 \%$ glucose log444 phase growth. Proteins on right are increased in whole-cell abundance with 4hrs AGR, those of

445 left decreased in abundance. Data are average of 4 independent expts. F) Model depicting TG 446 lipolysis driven LCL-LD formation, and resulting changes in LD translocation to ER network 447 targeting. Scale bars $5 \mu \mathrm{m}$.

\section{Supplemental Figure Legends:}

450 Supplemental Figure 1: LD lipid phase transitions characterized by cryo-FIB and cryo-ET.

451 A-J) Electron dose series ("bubblegrams") for LDs from cryo-FIB milled WT yeast in log phase

452 (A-E) or after AGR (F-J); series of 2D cryo-EM images were recorded of the same LDs exposed 453 to increasing electron dose (1 - 400 e-/Å2). Note that liquid-crystalline layers (LCLs) (see box in 454 G magnified in $\mathbf{J}$ ) and excessive bubbling in LD centers (starting at an electron dose $<30$ e-/ $\AA^{2}$ ) 455 occurred only under AGR. Even at 400 e-/ $\AA^{2}$ electron dose, minimal bubbling (white arrowheads 456 in E).was observed in log WT. K-M) Representative tomographic slices from cryo-FIB-milled 457 and cryo-ET reconstructed WT in log phase (K), nvj1. yeasts after 4hrs AGR (L), and 458 are 1are2 $\triangle$ yeast after $4 \mathrm{hrs}$ AGR $(\mathbf{M})$. The nucleus-vacuole junction (black arrowheads in $\mathbf{K}$ and 459 M) was observed in WT and are1are2 $\Delta$ yeasts, but absent in nvj1 $\Delta$ yeast (white arrowhead in 460 L). No LDs were found in are1are2 $\Delta$ yeast. V, vacuole. N, nucleus. L, lipid droplet, $M$, 461 mitochondrion. N) Yeast stained with LD marker MDH in log and 4hrs AGR. Scale bars: 50nm 462 (A-I), 200nm (K-M), 25nm (J).

464 Supplemental Figure 2: Erg6 LD targeting is influenced by AGR-associated LCL-LD 465 formation. A) WT or tg/3,4,5 Erg6-GFP yeast in log or AGR conditions. B) Erg6-DsRed2 466 localized to LDs in log and 4hrs AGR. Scale bars $5 \mu \mathrm{m}$.

468 Supplemental Figure 3: Selective delocalization of LD proteins during AGR stress. A) 469 Yeast expressing mNg-tagged Ayr1 and Anr2 and stained with LD marker MDH in log-phase or 470 4hrs AGR conditions. B) Yeast expressing Tgl5-mNg with MDH LD stain. Scale bars $5 \mu \mathrm{m}$. 
472 Supplemental Figure 4: Additional LD proteins examined in log-phase and AGR

473 conditions. A) Heat map depicting relative \% changes in annotated LD proteins from log to

474 4hrs AGR conditions. Average of 4 independent log-phase and 4hrs AGR experiments. B)

475 Yeast with $\mathrm{mNg}$-tagged Iml2 or Gvp36 and stained with $\mathrm{MDH}$ in log-phase and 4hrs AGR

476 conditions. Scale bars $5 \mu \mathrm{m}$.

477

478 Supplemental Movie 1: Tomographic reconstruction of a cryo-FIB-milled WT yeast cell after 479 4hrs acute glucose restriction. Compare with Figure 1A. Scale bar: 200nm.

Supplemental Movie 2: Tomographic reconstruction of a LD from a cryo-FIB-milled WT yeast cell in log phase (grown with $2 \%$ glucose). Compare with Figure 1B. Scale bar: $50 \mathrm{~nm}$.

Supplemental Movie 3: Tomographic reconstruction of a LD from a cryo-FIB-milled WT yeast cell after 4hrs AGR. Compare with Figure 1C. Scale bar: 50nm.

\section{References}

Aronova, M.A., Sousa, A.A., and Leapman, R.D. (2011). EELS characterization of radiolytic products in frozen samples. Micron (Oxford, England : 1993) 42, 252-256.

490 Bersuker, K., Peterson, C.W.H., To, M., Sahl, S.J., Savikhin, V., Grossman, E.A., Nomura, D.K., 491 and Olzmann, J.A. (2018). A Proximity Labeling Strategy Provides Insights into the Composition 492 and Dynamics of Lipid Droplet Proteomes. Developmental cell 44, 97-112 e117.

493 Caillon, L., Nieto, V., Gehan, P., Omrane, M., Rodriguez, N., Monticelli, L., and Thiam, A.R. 494 (2020). Triacylglycerols sequester monotopic membrane proteins to lipid droplets. Nature 495 communications 11, 3944.

496 Chorlay, A., and Thiam, A.R. (2020). Neutral lipids regulate amphipathic helix affinity for model 497 lipid droplets. The Journal of cell biology 219.

498 Currie, E., Guo, X., Christiano, R., Chitraju, C., Kory, N., Harrison, K., Haas, J., Walther, T.C., 499 and Farese, R.V. (2014). High confidence proteomic analysis of yeast LDs identifies additional 500 droplet proteins and reveals connections to dolichol synthesis and sterol acetylation[S]. Journal 501 of lipid research 55, 1465-1477. 
502 Czabany, T., Wagner, A., Zweytick, D., Lohner, K., Leitner, E., Ingolic, E., and Daum, G. (2008).

503 Structural and biochemical properties of lipid particles from the yeast Saccharomyces 504 cerevisiae. The Journal of biological chemistry 283, 17065-17074.

505 Eisenberg, T., and Büttner, S. (2014). Lipids and cell death in yeast. FEMS Yeast Res 14, 179506197.

507 Engelman, D.M., and Hillman, G.M. (1976). Molecular organization of the cholesteryl ester 508 droplets in the fatty streaks of human aorta. The Journal of clinical investigation 58, 997-1007.

509 Gao, Q., Binns, D.D., Kinch, L.N., Grishin, N.V., Ortiz, N., Chen, X., and Goodman, J.M. (2017). 510 Pet10p is a yeast perilipin that stabilizes lipid droplets and promotes their assembly. Journal of 511 Cell Biology 216, 3199-3217.

512 Giménez-Andrés, M., Emeršič, T., Antoine-Bally, S., D'Ambrosio, J.M., Antonny, B., Derganc, J., 513 and Čopič, A. (2021). Exceptional stability of a perilipin on lipid droplets depends on its polar 514 residues, suggesting multimeric assembly. eLife 10, e61401.

515 Ginsburg, G.S., Walsh, M.T., Small, D.M., and Atkinson, D. (1984). Reassembled plasma low 516 density lipoproteins. Phospholipid-cholesterol ester-apoprotein B complexes. The Journal of 517 biological chemistry 259, 6667-6673.

518 Hariri, H., Rogers, S., Ugrankar, R., Liu, Y.L., Feathers, J.R., and Henne, W.M. (2018). Lipid 519 droplet biogenesis is spatially coordinated at ER-vacuole contacts under nutritional stress. 520 EMBO reports 19, 57-72.

521 Jacquier, N., Choudhary, V., Mari, M., Toulmay, A., Reggiori, F., and Schneiter, R. (2011). Lipid 522 droplets are functionally connected to the endoplasmic reticulum in Saccharomyces cerevisiae. 523 Journal of cell science 124, 2424-2437.

524 Kassan, A., Herms, A., Fernandez-Vidal, A., Bosch, M., Schieber, N.L., Reddy, B.J., Fajardo, 525 A., Gelabert-Baldrich, M., Tebar, F., Enrich, C., et al. (2013). Acyl-CoA synthetase 3 promotes 526 lipid droplet biogenesis in ER microdomains. The Journal of cell biology 203, 985-1001.

527 Katz, S.S., Small, D.M., Brook, J.G., and Lees, R.S. (1977). The storage lipids in Tangier 528 disease. A physical chemical study. The Journal of clinical investigation 59, 1045-1054. 
529 Kroon, P.A. (1981). The order-disorder transition of the core cholesteryl esters of human plasma 530 low density lipoprotein. A proton nuclear magnetic resonance study. The Journal of biological 531 chemistry $256,5332-5339$.

532 Lang, M.J., Martinez-Marquez, J.Y., Prosser, D.C., Ganser, L.R., Buelto, D., Wendland, B., and 533 Duncan, M.C. (2014). Glucose starvation inhibits autophagy via vacuolar hydrolysis and induces 534 plasma membrane internalization by down-regulating recycling. The Journal of biological 535 chemistry 289, 16736-16747.

536 Leapman, R.D., and Sun, S. (1995). Cryo-electron energy loss spectroscopy: observations on 537 vitrified hydrated specimens and radiation damage. Ultramicroscopy 59, 71-79.

538 Leber, R., Landl, K., Zinser, E., Ahorn, H., Spök, A., Kohlwein, S.D., Turnowsky, F., and Daum, 539 G. (1998). Dual localization of squalene epoxidase, Erg1p, in yeast reflects a relationship 540 between the endoplasmic reticulum and lipid particles. Molecular biology of the cell 9, 375-386.

541 Leber, R., Zinser, E., Zellnig, G., Paltauf, F., and Daum, G. (1994). Characterization of lipid 542 particles of the yeast, Saccharomyces cerevisiae. Yeast (Chichester, England) 10, 1421-1428.

543 Lucken-Ardjomande Häsler, S., Vallis, Y., Jolin, H.E., McKenzie, A.N., and McMahon, H.T. 544 (2014). GRAF1a is a brain-specific protein that promotes lipid droplet clustering and growth, and 545 is enriched at lipid droplet junctions. Journal of cell science 127, 4602-4619.

546 Lundberg, B. (1985). Chemical composition and physical state of lipid deposits in 547 atherosclerosis. Atherosclerosis 56, 93-110.

548 Mahamid, J., Tegunov, D., Maiser, A., Arnold, J., Leonhardt, H., Plitzko, J.M., and Baumeister, 549 W. (2019). Liquid-crystalline phase transitions in lipid droplets are related to cellular states and 550 specific organelle association. Proceedings of the National Academy of Sciences 116, 1686655116871.

552 Marini, G., Nüske, E., Leng, W., Alberti, S., and Pigino, G. (2020). Reorganization of budding 553 yeast cytoplasm upon energy depletion. Molecular biology of the cell 31, 1232-1245.

554 Moldavski, O., Amen, T., Levin-Zaidman, S., Eisenstein, M., Rogachev, I., Brandis, A., 555 Kaganovich, D., and Schuldiner, M. (2015). Lipid Droplets Are Essential for Efficient Clearance 556 of Cytosolic Inclusion Bodies. Developmental cell 33, 603-610. 
557 Ohsaki, Y., Cheng, J., Fujita, A., Tokumoto, T., and Fujimoto, T. (2006). Cytoplasmic lipid 558 droplets are sites of convergence of proteasomal and autophagic degradation of apolipoprotein 559 B. Molecular biology of the cell 17, 2674-2683.

560 Olarte, M.J., Kim, S., Sharp, M.E., Swanson, J.M.J., Farese, R.V., Jr., and Walther, T.C. (2020). 561 Determinants of Endoplasmic Reticulum-to-Lipid Droplet Protein Targeting. Developmental cell $56254,471-487 . e 477$.

563 Olzmann, J.A., and Carvalho, P. (2019). Dynamics and functions of lipid droplets. Nature 564 reviews Molecular cell biology 20, 137-155.

565 Rogers, S., Hariri, H., Wood, N.E., Speer, N.O., and Henne, W.M. (2021). Glucose restriction 566 drives spatial reorganization of mevalonate metabolism. eLife 10.

567 Santinho, A., Chorlay, A., Foret, L., and Thiam, A.R. (2021). Fat inclusions strongly alter 568 membrane mechanics. Biophysical journal 120, 607-617.

569 Schmidt, C., Athenstaedt, K., Koch, B., Ploier, B., and Daum, G. (2013). Regulation of the yeast 570 triacylglycerol lipase TGI3p by formation of nonpolar lipids. The Journal of biological chemistry $571288,19939-19948$.

572 Shimobayashi S, O.Y. (2019). Universal phase behaviors of intracellular lipid droplets. 573 Proceedings of the National Academy of Sciences of the United States of America 116, 2544057425445.

575 Walther, T.C., Chung, J., and Farese, R.V., Jr. (2017). Lipid Droplet Biogenesis. Annual review 576 of cell and developmental biology 33, 491-510.

577 Wang, H., Becuwe, M., Housden, B.E., Chitraju, C., Porras, A.J., Graham, M.M., Liu, X.N., 578 Thiam, A.R., Savage, D.B., Agarwal, A.K., et al. (2016). Seipin is required for converting 579 nascent to mature lipid droplets. eLife 5.

580 Welte, M.A., and Gould, A.P. (2017). Lipid droplet functions beyond energy storage. Biochimica 581 et biophysica acta Molecular and cell biology of lipids 1862, 1260-1272.

582 Wilfling, F., Wang, H., Haas, J.T., Krahmer, N., Gould, T.J., Uchida, A., Cheng, J.X., Graham, 583 M., Christiano, R., Fröhlich, F., et al. (2013). Triacylglycerol synthesis enzymes mediate lipid 584 droplet growth by relocalizing from the ER to lipid droplets. Developmental cell 24, 384-399. 
585 Wood, N.E., Kositangool, P., Hariri, H., Marchand, A.J., and Henne, W.M. (2020). Nutrient 586 Signaling, Stress Response, and Inter-organelle Communication Are Non-canonical 587 Determinants of Cell Fate. Cell reports 33, 108446. 

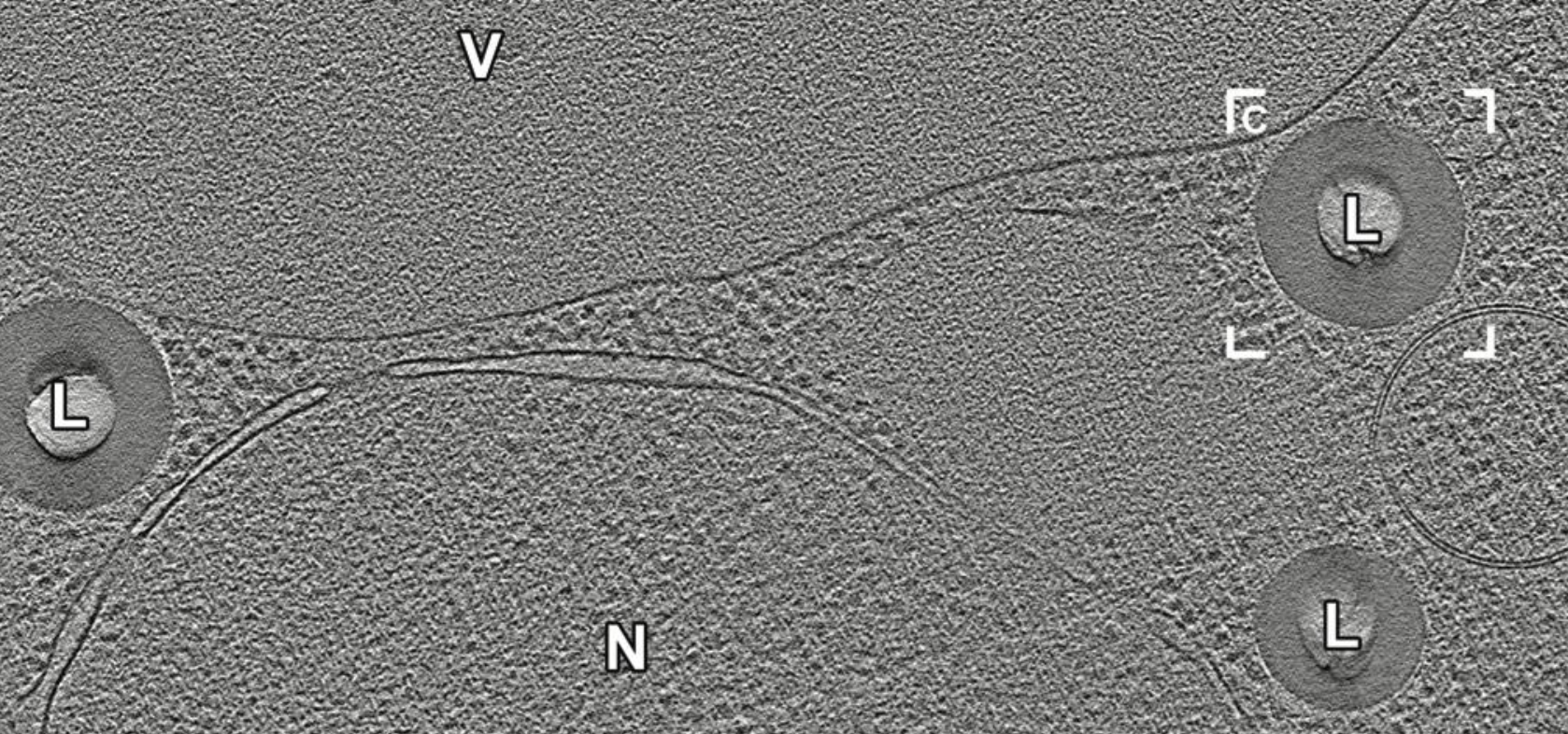

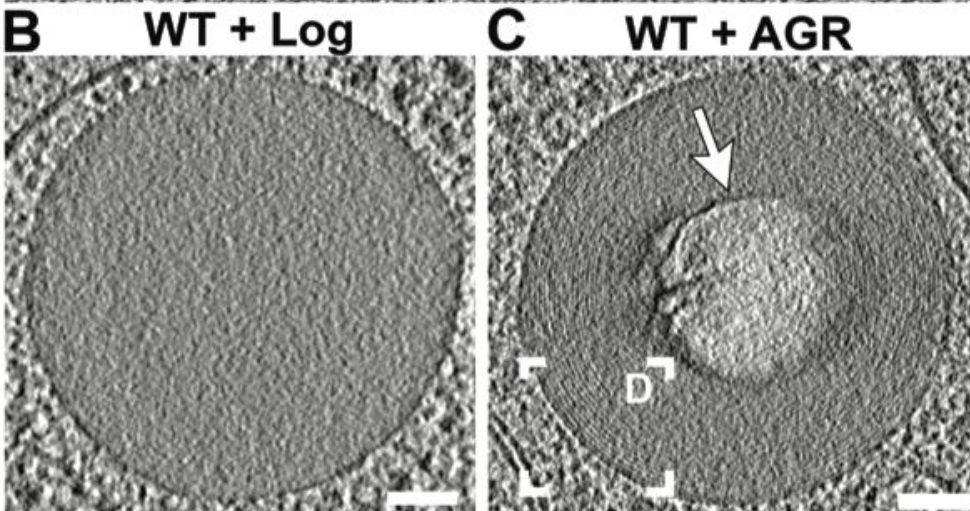

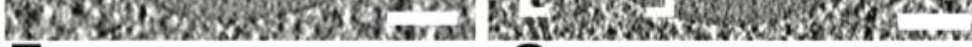

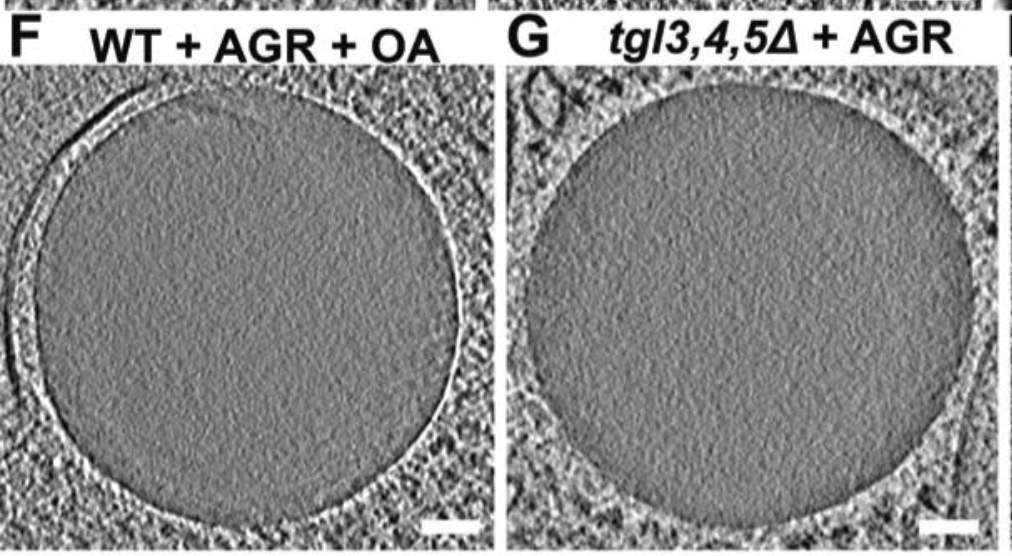

K

Quantification of neutral lipids

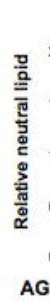

M

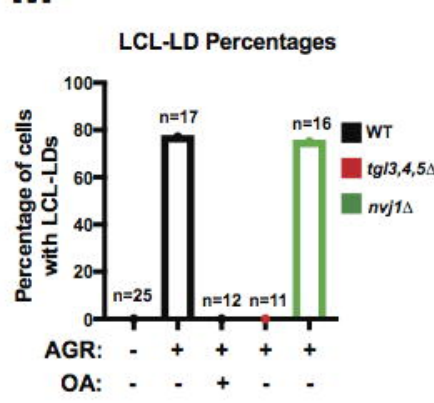

D $E$

Ehite

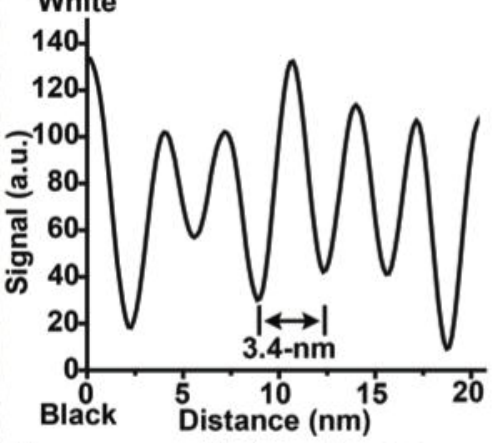

$n v j 1 \Delta+A G R$

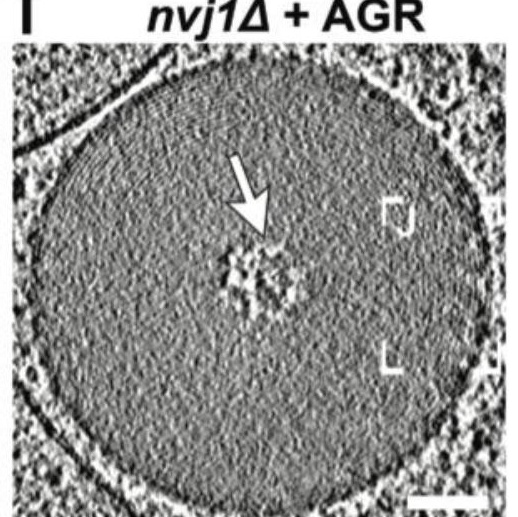

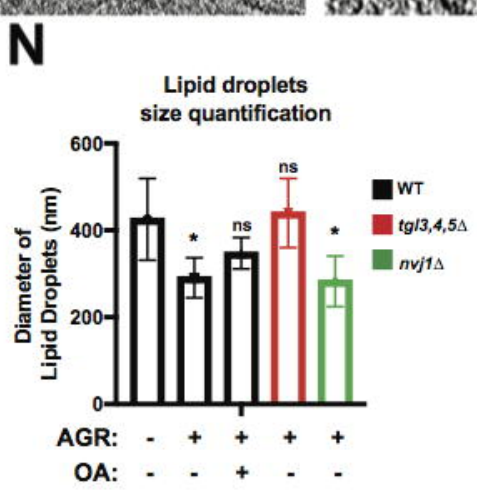

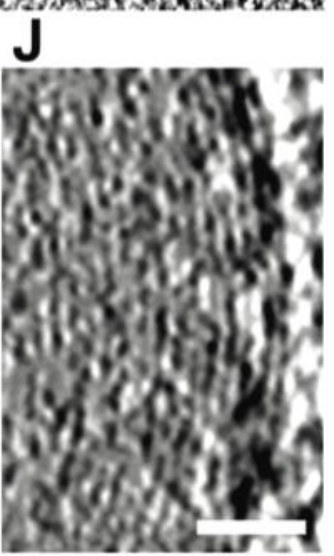




\section{bioRxiv preprint doi: https://doi.org/10.1101/2021.08.30.458229; this version posted August 31, 2021. The copyright holder for this preprint}

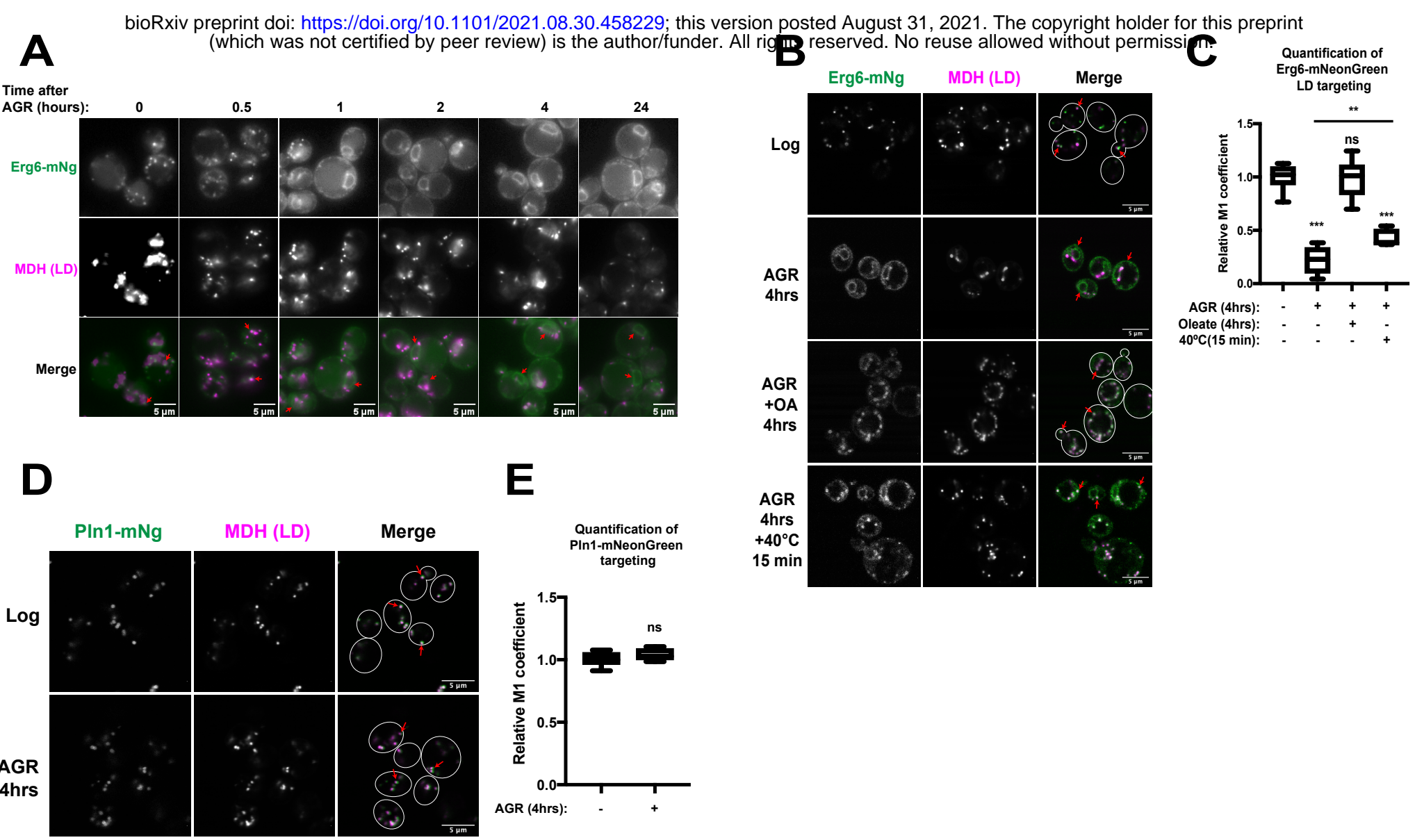


bioRxiv preprint doi: https://doi.org/10.1101/2021.08.30.458229; this version posted August 31, 2021. The copyright holder for this preprint A (which was not certified by peer review) is the author/funder. All right $\mathbf{B}^{\text {served. No reuse allowed without permission. }}$

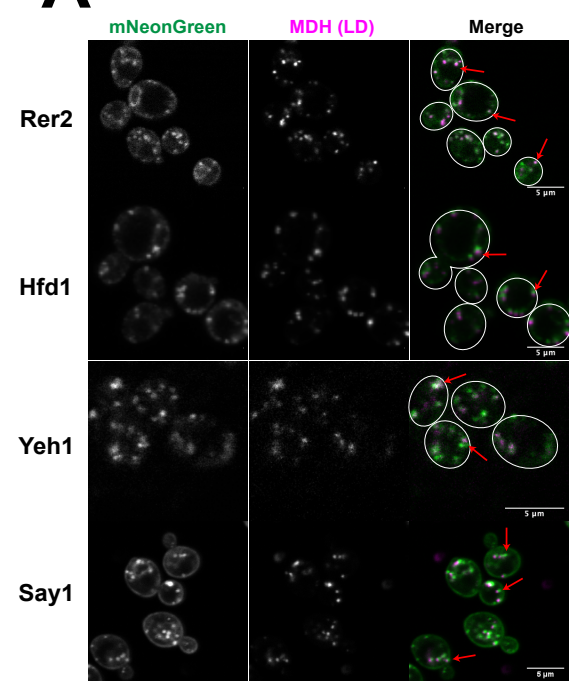

Log

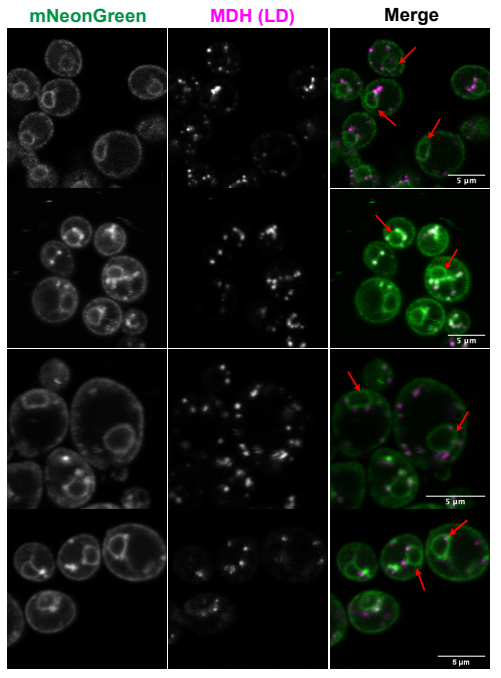

AGR
Quantification of protein targeting

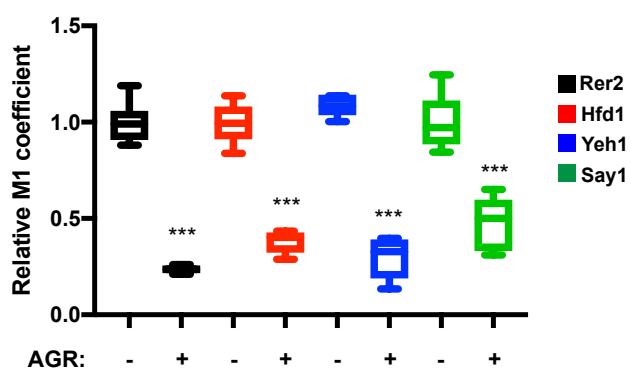

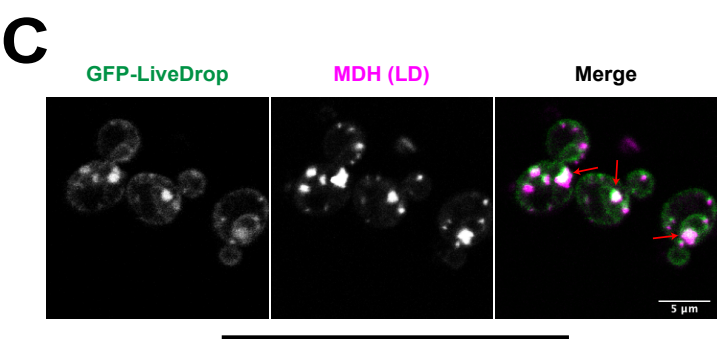

$\log$
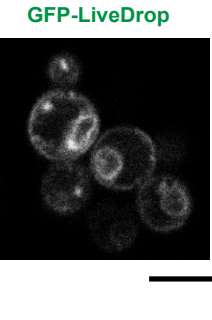

AGR

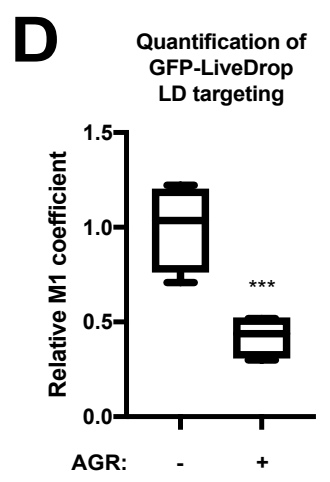

$E$

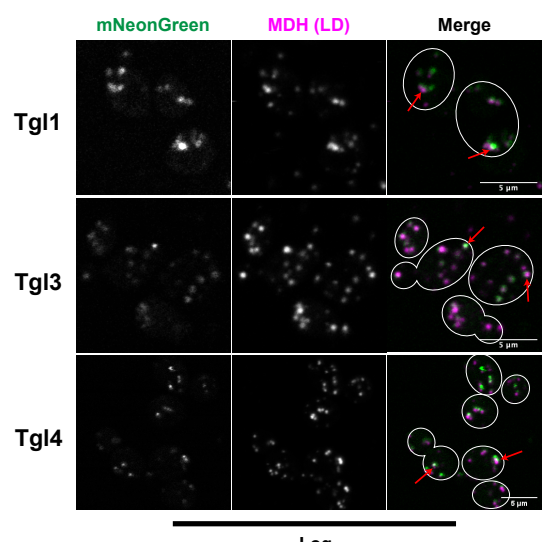

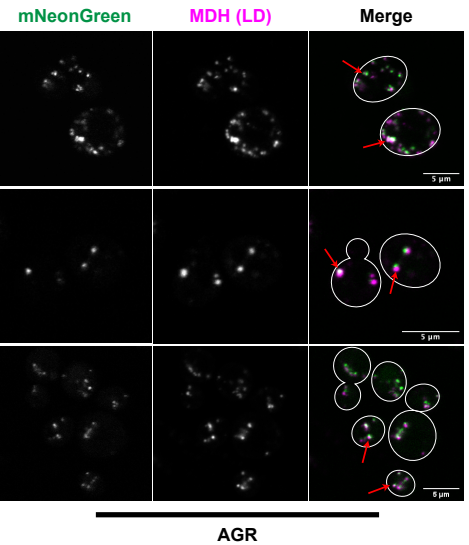

F

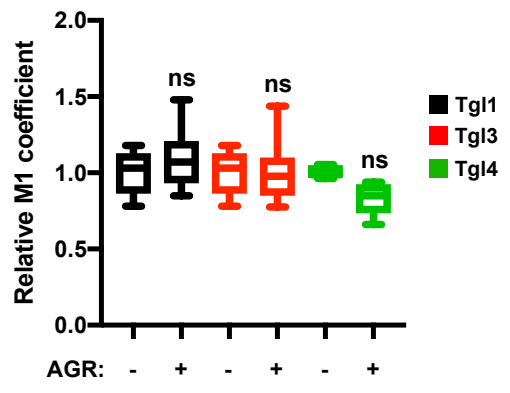


(which was not certified by peer review) is the author/funder. All rights reserved No reuse allowed wjthout permission.

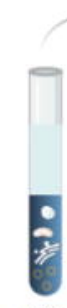

wCL

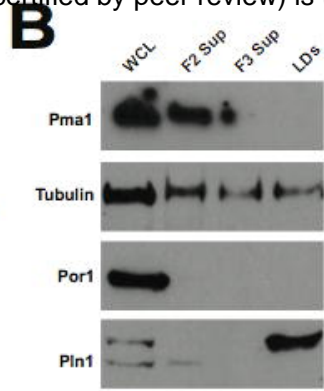

Percent of
C

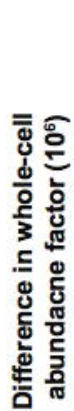
and LD-fraction proteome during AGR

D
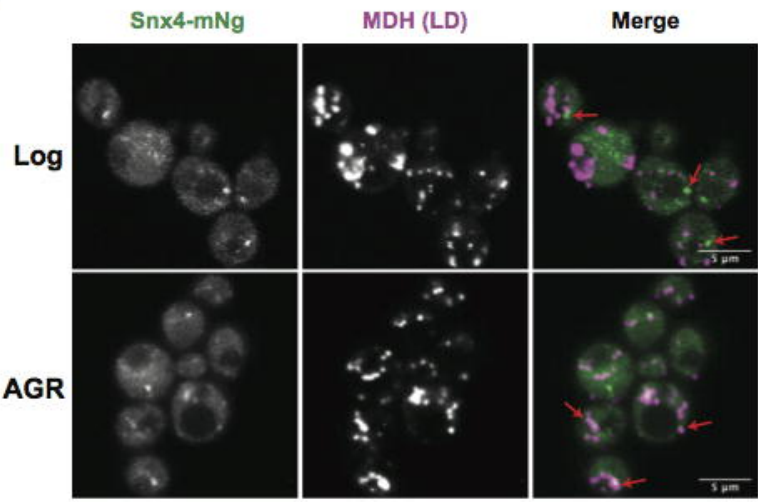

Quantification of whole-cell proteome changes during log to AGR transition

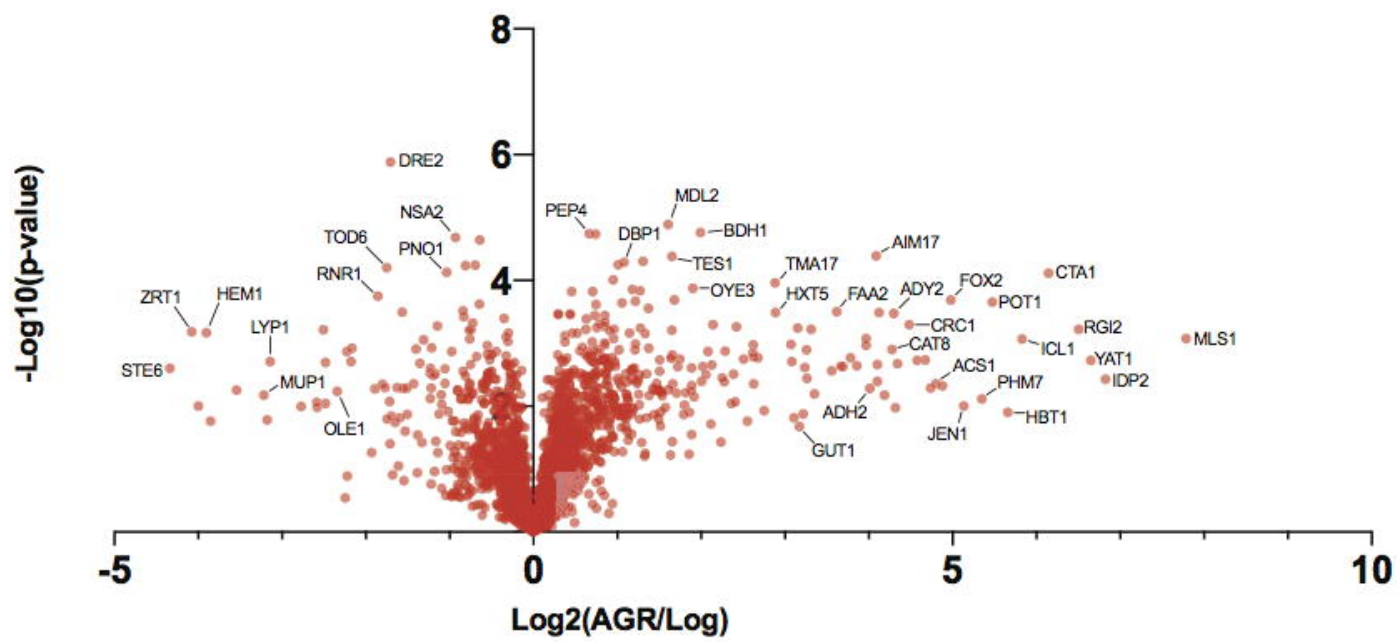

$\mathbf{F}$

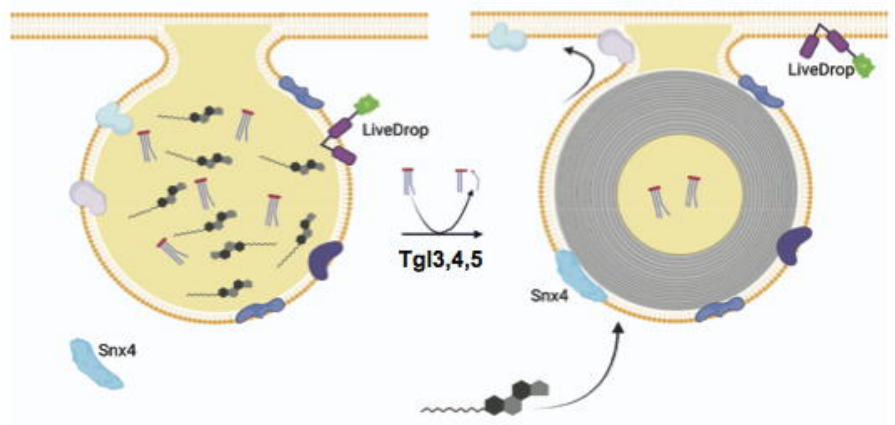

Log (amorphous LD core)

AGR (liquid-crystalline LD core) 